\title{
Topological Invariants of Connections on Symplectic Manifolds
}

\section{E. Tamarkin}

UDC 514.7

\section{§1. Introduction}

Let $M^{2 n}$ be a $2 n$-dimensional symplectic manifold, let $E \rightarrow M$ be a vector bundle whose structure group is a connected semisimple Lie group $G$, let $\nabla^{M}$ be a symplectic connection on $M$, and let $\nabla^{E}$ be a connection on $E$.

This paper is devoted to the problem of finding all closed differential forms on $M$ that can be written in local Darboux coordinates as polynomials in finite-order derivatives of the coefficients of the connections $\nabla^{E}$ and $\nabla^{M}$ on condition that the cohomology class of the manifold $M$ defined by such a form is preserved under the deformations of the connections. It is required that this differential form could be well defined on $M$. This is possible only for the case in which the dependence of this form on the connection coefficients is preserved under the transformations of Darboux coordinates. These forms are said to be invariant.

A similar problem for Riemannian manifolds was solved by Abramov [1] (also see Gilkey [2]). For a more detailed statement of this problem and its solution see Atiyah, Bott, and Patody [3].

By analogy with the case of Riemannian manifolds, every invariant form on $M$ is a polynomial in Pontryagin classes of the manifold $M$, characteristic classes of the bundle $E$, and the symplectic form $\omega$ (up to the so-called trivial forms whose cohomology classes are always trivial).

The author thanks B. V. Fedosov for the statement of the problem and constant attention and B. L. Feigin for valuable advice.

\section{§2. Statement of the Problem}

Let $U \subset \mathbb{R}^{2 n}$ be the ball $\sum_{i=1}^{2 n} x_{i}^{2}<1$, let $\omega=\sum_{i=1}^{n} d x^{i} \wedge d x^{n+i}$ be a symplectic form, let $\langle$, $\rangle$ be the corresponding inner product, let $\left\langle d x^{i}, d x^{n+i}\right\rangle=-1$, and let $\partial$ be the connection on $T^{*} U$ defined by the conditions $\partial d x^{i}=0, i=1, \ldots, 2 n$ (in other words; $\partial$ is the ordinary differential).

Every torsion-free connection $\nabla$ on $T^{*} U$ preserving the form $\omega$ (i.e., a symplectic connection) is determined by a tensor $\Gamma \in C^{\infty}\left(U, S^{3} T^{*} U\right)$ as follows:

$$
\nabla \eta=\partial \eta+\langle\Gamma, \eta\rangle, \quad \eta \in C^{\infty}\left(U, T^{*} U\right)
$$

Let $E=G \times U \rightarrow U$ be a principal bundle whose structure group is a semisimple Lie group $G$ with Lie algebra $\mathfrak{g}$. Every connection $\nabla^{E}$ on $G \times U$ is determined by a 1 -form $\vartheta \in C^{\infty}\left(U, T^{*} U\right) \otimes \mathfrak{g}$.

Let $V$ be an open subset of $U$. Denote by $\mathcal{A}_{V}$ the polynomial algebra in the variables $\vartheta$ and $\Gamma$ and their partial derivatives with respect to $x$ with coefficients belonging to $C^{\infty}(\bar{V})$ (the bar denotes the closure). Each element $\varphi \in \mathcal{A}_{V}$ determines a functional $\varphi[\vartheta, \Gamma] \in C^{\infty}(\bar{V})$ of $\vartheta$ and $\Gamma$.

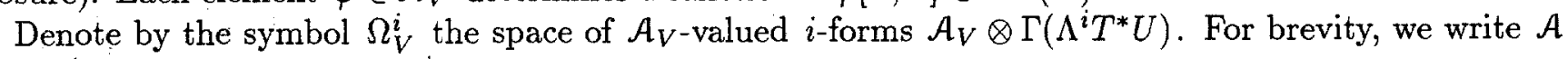
and $\Omega^{i}$ instead of $\mathcal{A}_{U}$ and $\Omega_{U}^{i}$, respectively.

Let $\varphi \in \Omega_{V}^{i}$ and let $\varphi=\sum_{\mu} \varphi_{\mu} d x^{\mu}$. We set

$$
\varphi[\vartheta, \Gamma]=\sum_{\mu} \varphi_{\mu}[\vartheta, \Gamma] d x^{\mu}
$$

We must find conditions under which the form $\varphi \in \Omega^{i}$ does not depend on the choice of Darboux coordinates and on the trivialization of $E$.

Let $V_{1}$ and $V_{2}$ be open in $U$, let $s: V_{1} \rightarrow V_{2}$ be a diffeomorphism preserving $\omega$, and let $h: V_{1} \rightarrow G$ be an arbitrary smooth mapping. Let us define a morphism of the bundles $\sigma: G \times V_{1} \rightarrow G \times V_{2}$ by the formula

$$
\sigma(g, x)=(g \cdot h(x), s(x)), \quad g \in G, x \in V_{1} .
$$

Moscow Institute of Physics and Engineering (MFTI). Translated from Funktsional'nyi Analiz i Ego Prilozheniya, Vol. 29, No. 4, pp. 45-56, October-December, 1995. Original article submitted June 1, 1994. 
For brevity, we write transformations (1) in the form $\sigma=(s, h)$.

The connections $s^{*} \nabla$ and $\sigma^{*} \nabla^{E}$ are defined on $V_{1}$ and $G \times V_{1}$, respectively; therefore, the coefficients $s^{*} \Gamma$ and $\sigma^{*} \vartheta$ of these connections are also defined. We can construct a mapping $\sigma^{*}: \Omega_{V_{2}}^{i} \rightarrow \Omega_{V_{1}}^{i}$ as follows:

$$
\left(\sigma^{*} \varphi\right)[\vartheta, \Gamma]=s^{*} \varphi\left[\left(\sigma^{-1}\right)^{*} \vartheta,\left(\sigma^{-1}\right)^{*} \Gamma\right] .
$$

Definition 1. A form $\varphi \in \Omega^{i}$ is said to be globally defined if for each morphism (1) we have the relation

$$
\sigma^{*}\left(\varphi \mid V_{2}\right)=\left.\varphi\right|_{V_{1}}
$$

Denote by $D^{i}$ the space of globally defined $i$-forms.

Let $H$ be the Hamiltonian function with supp $H \subset U$, let $X_{H}$ be the corresponding Hamiltonian vector field, and let $\alpha: U \rightarrow \mathfrak{g}$ be an arbitrary smooth mapping. Let $p_{1}: G \times U \rightarrow U$ and $p_{2}: G \times U \rightarrow G$ be the projections onto the factors. We define a vector field $X_{\alpha, H}$ on $G \times U$ by the formulas

$$
\left.p_{1 *}\right|_{(g, x) \in G \times U} X_{\alpha, H}=\left.X_{H}\right|_{x},\left.\quad p_{2 *}\right|_{(g, x)} X_{\alpha, H}=L_{g *} \alpha(x),
$$

where $L_{g}$ is the left shift on $G$.

The fields $X_{\alpha, H}$ form a Lie algebra $\mathfrak{l}$ with the commutator

$$
\left[X_{\alpha_{1}, H_{1}}, X_{\alpha_{2}, H_{2}}\right]=X_{\left[\alpha_{1}, \alpha_{2}\right]_{\mathfrak{B}}+\left\langle d \alpha_{1}, d H_{2}\right\rangle-\left\langle d \alpha_{2}, d H_{1}\right\rangle,\left\langle d H_{1}, d H_{2}\right\rangle} \cdot
$$

The transformations $\sigma_{t}=\exp \left(t X_{\alpha, H}\right)$ are of the form (1). Let us define a representation $\delta$ of the Lie algebra $\mathfrak{l}$ on $\Omega^{i}$ as follows:

$$
\delta \in \Omega^{i} \otimes l^{*}, \quad \delta_{X_{\alpha, H}} \varphi=d\left(\sigma_{t}^{*} \varphi\right) /\left.d t\right|_{t=0} .
$$

Proposition 1. A form $\varphi$ is globally defined if and only if $\delta \varphi=0$.

Proof. The necessity is clear. Let us prove the sufficiency.

First of all, we note that if $\sigma_{t}=\exp \left(t X_{\alpha, H}\right)$, then $d \sigma_{t}^{*} \varphi / d t=\sigma_{t}^{*} \delta_{X_{\alpha, H}} \varphi=0$ and hence $\sigma_{t}^{*} \varphi=0$.

Let $\delta \varphi=0$ and let $\sigma=(s, h)$ be defined by (1). The transformation $\sigma$ is factorized as a product of two transformations: $\sigma=\sigma_{1} \circ \tilde{s}$, where $\sigma_{1}=\left(I, h\left(s^{-1}(x)\right)\right)$ and $\tilde{s}=\left(s, 1_{G}\right)$. Since the set $U$ is contractible, for some smooth function $\alpha: U \rightarrow \mathfrak{g}$ we have $h\left(s^{-1}(x)\right)=\exp \alpha(x)$ and $\sigma_{1}=\exp \left(X_{\alpha, 0}\right)$. Thus, $\sigma^{*} \varphi=\tilde{s}^{*} \sigma_{1}^{*} \varphi=\tilde{s}^{*} \varphi$. Since the operation $\tilde{s}^{*}$ is local, it suffices to prove that for each $x_{0} \in V_{1}$ there exists a small ball $B=B\left(x_{0}, \varepsilon\right): \sum\left(x_{i}-x_{0 i}\right)^{2}=\varepsilon^{2}$ for which $\tilde{s}^{*}\left(\left.\varphi\right|_{s(B)}\right)=\left.\varphi\right|_{B}$.

Without loss of generality, we can assume that $\bar{B}, \overline{s(B)} \subset U$. Let $B_{0}$ be a closed ball that contains $\bar{B}$ and $\overline{s(B)}$. The transformation $\tilde{s}$ on $U$ can be factorized as a product $\tilde{s}=\left(s_{2}, 0\right) \circ\left(s_{1}, 0\right)$, where $\left(s_{2}, 0\right)=\exp X_{0, H}$ and the function $H$ is such that $H=\sum_{i} a_{i} x_{i}+\sum_{i, j} a_{i j} x_{i} x_{j}$ for $x \in B_{0}$ and the constants $a_{i}, a_{i j}$ are chosen to satisfy $s_{2}\left(x_{0}\right)=s_{1}\left(x_{0}\right)$ and $J s_{2}\left(x_{0}\right)=J s_{1}\left(x_{0}\right)$, where $J$ is the Jacobi matrix of a differentiable mapping. This choice is possible because $J_{\mathcal{S}_{1}}\left(x_{0}\right)$ is a symplectic matrix and, hence, it is representable in the form $\exp \left(\omega^{i s} a_{s j}\right)$, where $a_{s j}$ is a symmetric matrix. We obtain $s_{1}\left(x_{0}\right)=x_{0}, J s_{3}\left(x_{0}\right)=I$, and $\tilde{s}^{*} \varphi=\left(1, s_{1}\right)^{*} \varphi$.

Let $p^{i}=x^{i}$ and $q^{i}=x^{n+i}, i=1, \ldots, n$. The transformation $s_{1}$ can be defined in a sufficiently small ball $\widetilde{B}$ by means of the generating function $S$ :

$$
\tilde{p}=\partial S(p, \tilde{q}) / \partial \tilde{q}, \quad q=\partial S(p, \tilde{q}) / \partial p
$$

where $s_{1}(p, q)=(\tilde{p}, \tilde{q})$ and

$$
S(p, \tilde{q})=\sum_{i} p^{i} \tilde{q}^{i}+o\left(\sum_{i}\left(p^{i}\right)^{2}+\left(\tilde{q}^{i}\right)^{2}\right) .
$$

Choose a ball $\widetilde{B}$ such that the family of functions

$$
S^{t}=t \sum_{i} p^{i} \tilde{q}^{i}+(1-t) S \text { for } 0 \leq t \leq 1
$$


defines a family of transformations $s^{t}: \widetilde{B} \rightarrow U$ by formula $(*)$. We can suppose that $\overline{s^{t}(\widetilde{B})} \subset U$.

Let $H^{t}$ be a family of Hamiltonian functions satisfying the relation

$$
X_{H^{t}}(x)=d s^{t}(x) / d t, \quad x \in s^{t}(\widetilde{B}) .
$$

This family exists because $s^{t}(\widetilde{B})$ is a contractible set.

We have

$$
d\left(s^{t}, 0\right)^{*} \varphi / d t=s^{t *} \delta_{X_{0, H^{t}}} \varphi=0 .
$$

Thus, $s_{1}^{*} \varphi=\left(s^{0}, 0\right)^{*} \varphi=\left(s^{1}, 0\right)^{*} \varphi=\varphi$ as desired.

We set

$$
\delta_{X_{\alpha, H}} \Gamma=\left.\frac{d}{d t} s_{t}^{*} \Gamma\right|_{t=0}, \quad \delta_{X_{\alpha, H}} \vartheta=\left.\frac{d}{d t} \sigma_{t}^{*} \vartheta\right|_{t=0},
$$

where $s_{t}=\exp \left(t X_{H}\right)$. Then

$$
\delta_{X_{\alpha, H}} \Gamma=L_{X_{H}} \Gamma-\partial^{3} H
$$

where $\partial^{3} H=\partial \partial d H \in C^{\infty}\left(U, S^{3} T^{*} U\right)$; moreover,

$$
\begin{aligned}
\delta_{X_{\alpha, H}} \vartheta & =L_{X_{H}} \vartheta-d \alpha-[\vartheta, \alpha], \\
\left(\delta_{X_{\alpha, H}} \varphi\right)[\vartheta, \Gamma] & =L_{X_{H}} \varphi[\vartheta, \Gamma]-\left.\frac{d}{d t} \varphi\left[\vartheta+t \delta_{X_{\alpha, H}} \vartheta, \Gamma+t \delta_{X_{\alpha, H}} \Gamma\right]\right|_{t=0},
\end{aligned}
$$

where $L_{X_{H}} \Gamma$ is the Lie derivative of the tensor on $T^{*} U$.

Let us pass directly to the statement of the problem.

Definition 2. A form $\varphi \in D^{k}$ is said to be trivial if $\varphi=d \eta$ and $\eta \in D^{k-1}$.

Definition 3. A form $\varphi \in D^{k}$ is said to be invariant if

1) $d \varphi=0$,

2) for $k=2 n, \Gamma=\left.\bar{\Gamma}\right|_{\partial U}$, and $\vartheta=\left.\bar{\vartheta}\right|_{\partial U}$ we have $\int_{U} \varphi[\vartheta, \Gamma]=\int_{U} \varphi[\bar{\vartheta}, \bar{\Gamma}]$.

Condition 2 is equivalent to the fact that the Euler variational derivative of $\varphi$ with respect to $\vartheta$ and $\Gamma$ vanishes. This means $\left[5\right.$, p. 434] that $\varphi=d \psi$, where $\psi \in \Omega^{2 n-1}$.

Our objective is to find all invariant forms up to trivial ones; in other words, we have to find the cohomology of the complex

$$
\tilde{D}^{\cdot}: 0 \rightarrow D^{0} \stackrel{d}{\rightarrow} D^{1} \stackrel{d}{\rightarrow} \ldots \stackrel{d}{\rightarrow} D^{2 n-1} \stackrel{d}{\rightarrow} D^{2 n} \cap d\left(\Omega^{2 n-1}\right) \rightarrow 0 .
$$

Now we can state the result. Let

$$
R \in \mathfrak{s p}(2 n) \otimes C^{\infty}\left(U, \Lambda^{2} T^{*} U\right), \quad F \in \mathfrak{g} \otimes C^{\infty}\left(U, \Lambda^{2} T^{*} U\right)
$$

be the curvatures of the connections $\nabla$ and $\nabla^{E}$, respectively.

Theorem. The cohomology classes of the complex $\tilde{D}$ are uniquely representable by the forms

$$
P(R \oplus F \oplus \omega),
$$

where $P$ is an arbitrary invariant polynomial on the Lie algebra $\mathfrak{s p}(2 n) \oplus \mathfrak{g} \oplus \mathbb{R}$.

The main tool used in the proof of this theorem is a bicomplex that is constructed in the next section.

\section{§3. Constructing a Bicomplex}

Consider an $\mathcal{A}$-module $\mathcal{V}$ that consists, by definition, of the expressions

$$
\psi[H, \alpha]=\sum_{|\mu| \leq N} a_{\mu} \partial_{\mu} H+\sum_{|\mu| \leq N ; \gamma} b_{\mu \gamma} \partial_{\mu} \alpha^{\gamma},
$$


where $\alpha=\sum \alpha^{\gamma} e^{\gamma}$ for some fixed basis $\left(e_{1}, e_{2}, \ldots\right)$ in $\mathfrak{g}$, and, as usual, for $\mu=\left(\mu_{1}, \ldots, \mu_{n}\right)$ we set $\partial_{\mu}=\partial_{\mu_{1}} \cdots \partial_{\mu_{n}}$ and $a_{\mu}, b_{\mu \gamma} \in \mathcal{A}$.

We write $A^{p, q}=\Lambda^{p} \mathcal{V} \otimes_{\mathcal{A}} \Omega^{q}$. Every element $\varphi \in A^{p, q}$ determines a multilinear skew-symmetric functional of $\left(\alpha_{1}, H_{1}\right), \ldots,\left(\alpha_{p}, H_{p}\right)$ :

$$
\varphi\left[\left(\alpha_{1}, H_{1}\right), \ldots,\left(\alpha_{p}, H_{p}\right)\right] \in \Omega^{q}
$$

Let $X_{\alpha_{i}, H_{i}} \in \mathfrak{l}$. We introduce the expression

$$
\varphi\left[X_{\alpha_{1}, H_{1}}, \ldots, X_{\alpha_{p}, H_{p}}\right]=\varphi\left[\left(\alpha_{1}, H_{1}\right), \ldots,\left(\alpha_{p}, H_{p}\right)\right] .
$$

Define an operator $\delta: A^{p, q} \rightarrow A^{p+1, q}$ by the formula

$$
\begin{aligned}
\delta \varphi\left(X_{1}, \ldots, X_{p+1}\right)= & \sum_{i}(-1)^{i+1} \delta_{X_{i}} \varphi\left(X_{1}, \ldots, \widehat{X}_{i}, \ldots, X_{p+1}\right) \\
& -\sum_{i, j}(-1)^{i+j+1} \varphi\left(\left[X_{i}, X_{j}\right], X_{1}, \ldots, \widehat{X}_{i}, \ldots, \widehat{X}_{j}, \ldots, X_{p+1}\right),
\end{aligned}
$$

where $X_{i} \in \mathfrak{l}$.

Formula (7) coincides with that for the differential in the complex of cochains of the Lie algebra $l$ with coefficients in the representation determined by $\delta$.

Introduce an operator $d: A^{p, q} \rightarrow A^{p, q+1}$ as follows:

$$
(d \varphi)\left(X_{1}, \ldots, X_{p}\right)=(-1)^{p} d\left(\varphi\left(X_{1}, \ldots, X_{p}\right)\right),
$$

where $d$ on the right-hand side is the ordinary exterior differential of a $q$-form.

We have a naturally defined exterior product $\wedge: A^{p, q} \otimes A^{r, s} \rightarrow A^{p+r, q+s}$. If $a \in A^{p, q}$ and $b \in A^{r, s}$, then $a \wedge b=(-1)^{(p+q)(r+s)} b \wedge a$.

Proposition 2. Let $a \in A^{p, q}$ and $b \in A^{r, s}$. Then the following assertions hold.

(1) $d(a \wedge b)=d a \wedge b+(-1)^{p+q} a \wedge d b$

(2) $\delta(a \wedge b)=\delta a \wedge b+(-1)^{p+q} a \wedge \delta b$

(3) $(d+\delta)^{2}=0$.

This means that $\left(A^{\cdot} \cdot \cdot, d, \delta\right)$ is a multiplicative bicomplex.

Proof. Assertions (1) and (2) directly follow from the definitions. By (1) and (2), it suffices to verify (3) only for $A^{1,0}$ and $A^{0,1}$. This proves the proposition.

In what follows we need the cohomology of the complex associated with the bicomplex $A^{*}, \cdot$. We calculate it by means of the spectral sequence of this bicomplex associated with the filtration $F^{p} A^{{ }^{*}}{ }^{\circ}=$ $\bigoplus_{m \geq p} A^{m, \cdot}$ (the differential $d$ in this spectral sequence maps upwards, and $\delta$ maps to the right).

Proposition 3. In the above spectral sequence we have $E_{1}^{q, p}=H^{q}\left(A^{p, \cdot}, d\right)=0$ for $p \neq 0$ and $q \neq 2 n$; $E_{1}^{q, 0}=0$ for $q \neq 0,2 n ;$ and, finally, $E_{1}^{0,0}=\mathbb{R}$. Hence, $H^{0}\left(A^{\cdot, \cdot}, d+\delta\right)=\mathbb{R}$ and $H^{i}\left(A^{\cdot, \cdot}, d+\delta\right)=0$, $0<i<2 n$.

Proof. We need the lemma below.

Denote by $A(p)$ the space of functionals of $p$ functions $u_{1}, \ldots, u_{p} \in C^{\infty}(\bar{U})$ of the form

$$
\varphi\left[u_{1}, \ldots, u_{p}\right]=\Phi\left(x, u_{i}, \partial_{\mu} u_{i}(|\mu| \leq N)\right) .
$$

We have the exterior differential $d: A(p) \otimes \Lambda^{k} \mathbb{R}^{2 n} \rightarrow A(p) \otimes \Lambda^{k+1} \mathbb{R}^{2 n}$,

$$
(d \varphi)\left[u_{1}, \ldots, u_{p}\right]=d\left(\varphi\left[u_{1}, \ldots, u_{p}\right]\right) .
$$

Lemma 1. The complex $\left(A(p) \otimes \Lambda^{\cdot} \mathbb{R}^{2 n}, d\right)$ is exact in all terms except for the zeroth and $2 n$ th. 
Proof. See [5, p. 434].

Let us find the cohomology of the complex $\left(A^{p, \cdot}, d\right)$ that is equal to $E_{1}^{\cdot, p}$ by definition. Let $\psi \in A^{p, q}$ and $d \psi=0, q \neq 0,2 n$. By Lemma 1 , we have $\psi=d \chi$, where $\chi$ is a smooth function of the variables

$$
\partial_{\mu} \vartheta, \partial_{\mu} \Gamma, \partial_{\mu} H_{i} \text {, and } \partial_{\mu} \alpha_{i} .
$$

Suppose that the degree of the polynomial $\psi$ in variables (8) is equal to $n$. Replace the function $\chi$ by its Taylor expansion in variables (8) up to the terms of order $n$ inclusive; denote this expansion by $\chi^{\prime}$. Clearly, $d \chi^{\prime}=\psi$ (this follows from the fact that $d$ maps every homogeneous polynomial in variables (8) to a homogeneous polynomial of the same degree).

Selecting the terms in $\chi^{\prime}$ that are multilinear with respect to $\left(\alpha_{i}, H_{i}\right)$ (i.e., the terms of the form

$$
\prod_{s} \partial_{\mu_{i_{s}}} \alpha_{i_{s}}^{\gamma_{s}} \prod_{t} \partial_{\mu_{j_{t}}} H_{j_{t}} \cdot \varsigma,
$$

where $i_{s}$ and $j_{t}$ range over $p$ pairwise distinct positive integers from 1 to $p$ and $\left.\varsigma \in \Lambda^{q-1} \mathbb{R}^{2 n}\right)$ and alternating with respect to $\left(\alpha_{i}, H_{i}\right)$, we obtain $\tilde{\chi} \in A^{p, q-1}$ and $d \widetilde{\chi}=\psi$. Thus, $E_{1}^{q, p}=0$ for $q>0$.

If $q=0$, then we see that the relation $d \psi=0$ implies $\psi=$ const $\in A^{0,0}$. Thus, $E_{1}^{p, 0}=0, p \neq 0$, and $E_{1}^{0,0}=\mathbb{R}$. This completes the proof of the proposition.

We now restate the condition of the problem in terms of the constructed bicomplex. Consider the complex $\left(D^{\cdot}, d\right)$ (the inclusion $d D^{i} \subset D^{i+1}$ is readily verified). We have a natural embedding $i: D^{*} \rightarrow$ $A^{0, \cdot}=\Omega^{*}$ that defines a morphism of the complex $D^{*}$ into the complex $\left(A^{*}, \cdot d+\delta\right)$ associated with the bicomplex $A^{*}$, . Denote this morphism by the same symbol $i$.

Proposition 4. The cohomology of the complex $\widetilde{D}^{\cdot}$ (complex (5)) coincides with the kernel of the induced cohomology mapping

$$
i_{*}: H^{\cdot}\left(D^{*}\right) \rightarrow H^{\cdot}\left(A^{\cdot, \cdot}, d+\delta\right) .
$$

Proof. For dimensions less than $2 n$ this follows from Proposition 3 and relation $H^{p}\left(\widetilde{D}^{*}\right)=H^{p}\left(D^{*}\right)$ for $p<2 n$.

Now let $p=2 n$ and let $\eta \in D^{2 n}$ be a representative of an element $\tilde{\eta} \in H^{2 n}\left(D^{*}\right)$. If $i_{*} \tilde{\eta}=0$, then $\eta=d \chi$ and $\chi \in A^{0,2 n-1}$; hence, $\tilde{\eta} \in \widetilde{D}$ and $\tilde{\eta} \in H^{2 n}(\widetilde{D})$. Conversely, if $\eta \in D^{2 n} \cap d \Omega^{2 n-1}=\widetilde{D}^{2 n}$, then $i(\eta)=d \chi$ and $\chi \in A^{0,2 n-1}$. This means that the image of $\eta$ in $E_{1}^{2 n, 0}$ is zero. By Proposition 3, we have $E_{1}^{q, p}=0$ for $q+p=2 n$ and $q \neq 2 n$; therefore, the cohomology class $i(\eta)$ also vanishes.

This completes the proof of the proposition.

\section{§. Proof of the Theorem}

In this section our main tool is the multiplicative spectral sequence of the bicomplex $A^{\cdot, \cdot}$ associated with the filtration

$$
F^{q} A^{\cdot, \cdot}=\bigoplus_{r \geq q} A^{\cdot, r}
$$

(thus, the differential $\delta$ maps upwards, and $d$ maps to the right).

Let us restate the problem in terms of this spectral sequence. To this end, note that $D^{i}=E_{1}^{0, i}$. For the cohomology of the complex $D^{\cdot}$ we have $H^{i}\left(D^{*}\right)=E_{2}^{0, i}$. This follows from the well-known formulas $E_{1}^{p, q}=H^{p}\left(A^{*, q}, \delta\right)$ and $E_{2}^{p, q}=H^{q}\left(E_{1}^{p, \cdot}, d_{*}\right)$, where $d_{*}$ is the induced mapping. Therefore, the kernel of the mapping $i_{*}$ in Proposition 4 is equal to that of the mapping $E_{2}^{0, i} \rightarrow E_{\infty}^{0, i}$.

In other words, our objective is to find, in the terms $E_{2}^{0, i}$, the subspaces that are killed in the screening and prove that each element of this subspace can be represented in $E_{0}^{0, i}=\Omega^{i}$ by a unique element of the form (6) and each element of the form (6) represents an element of the indicated subspace.

Let us begin with calculating the term $E_{1}^{p, q}$. We denote by $\mathfrak{m}$ the Lie algebra $\mathfrak{g} \oplus \mathfrak{s p}(2 n) \oplus \mathbb{R}$.

Proposition 5. (1) $E_{1}^{p, q}=H^{p}\left(A^{*}, q, \delta\right) \cong H^{0}\left(A^{\cdot, q}\right) \otimes H^{p}(\mathrm{~m})$.

(2) There is an isomorphism of algebras $E_{1}^{, \cdot} \cong E_{1}^{0, \cdot} \otimes H^{\cdot}(\mathrm{m})$. 
Proof. Let $\Gamma=\Gamma_{i j k} d x^{i} \otimes d x^{j} \otimes d x^{k}$ and $\vartheta=\vartheta_{i \alpha} d x^{i} \otimes e^{\alpha}$, where $e^{\alpha}$ is a fixed basis in $g$. Let

$$
\begin{gathered}
R_{i j k l}=\partial \Gamma_{j k l} / \partial x^{i}-\partial \Gamma_{i k l} / \partial x^{j}+\omega^{p q}\left(\Gamma_{i p k} \Gamma_{j q l}-\Gamma_{j p k} \Gamma_{i q l}\right) \\
F_{i j \alpha} e^{\alpha}=\partial \vartheta_{j} / \partial x^{i}-\partial \vartheta_{i} / \partial x^{j}+\left[\vartheta_{i} \vartheta_{j}\right]_{\mathfrak{g}}
\end{gathered}
$$

be the curvatures of the connections $\nabla$ and $\nabla^{E}$.

Introduce the variables (here and below $I=\left(i_{1}, \ldots, i_{N}\right)$ )

$$
R_{i j k l ; I}=\nabla_{i_{1}} \ldots \nabla_{i_{N}} R_{i j k l}
$$

where $\nabla_{i_{k}}$ is the covariant derivative of a tensor on $T^{* \otimes} U$,

$$
F_{i j \alpha ; I} e^{\alpha}=\bar{\nabla}_{i_{1}} \ldots \bar{\nabla}_{i_{N}} F_{i j \alpha} e^{\alpha},
$$

where for $a \in T^{*} \otimes U$ and $b \in \mathfrak{g}$ we have $\bar{\nabla}_{i}(a \otimes b)=\nabla_{i} a \otimes b+a \otimes\left[\vartheta_{i}, b\right]+a \otimes\left(\partial b / \partial x^{i}\right)$, and

$$
\begin{aligned}
& r_{i j k l ; I}=\partial_{I}\left(\frac{\partial \Gamma_{j k l}}{\partial x^{i}}-\frac{\partial \Gamma_{i k l}}{\partial x^{j}}\right), \quad f_{i j \alpha ; I}=\partial_{I}\left(\frac{\partial \vartheta_{j \alpha}}{\partial x^{i}}-\frac{\partial \vartheta_{i \alpha}}{\partial x^{j}}\right), \\
& \gamma_{I}=\partial_{\left(i_{N} i_{N-1} \ldots i_{4}\right.} \Gamma_{\left.i_{3} i_{2} i_{1}\right)}, \quad N \geq 3, \quad \vartheta_{I \alpha}=\partial_{\left(i_{N} i_{N-1} \ldots i_{2}\right.} \vartheta_{\left.i_{1}\right) \alpha}, \quad N \geq 1 \text {, }
\end{aligned}
$$

where the parentheses symbolize symmetrization.

For brevity, we denote by $\mathcal{C}$ the algebra $C^{\infty}(\bar{U})$.

Introduce the linear spans

$$
\begin{aligned}
& A_{n}=\mathcal{C}\left\langle\partial_{I} \Gamma_{j k l}, \partial_{I} \vartheta_{i \alpha}(|I|=n)\right\rangle, \\
& B_{n}=\mathcal{C}\left\langle R_{i j k l ; I} ; F_{i j \alpha ; I}(|I|=n-1)\right\rangle, \\
& C_{n}=\mathcal{C}\left\langle\gamma_{I}(|I|=n+3), \vartheta_{J \alpha}(|J|=n+1)\right\rangle, \\
& \widetilde{B}_{n}=\mathcal{C}\left\langle r_{i j k l ; I} ; f_{i j \alpha ; I}(|I|=n-1)\right\rangle .
\end{aligned}
$$

We set $X^{n}=\bigoplus_{k=0}^{N} X_{k}$ for $X=A, B, C, \widetilde{B}$ and denote $X=\bigcup X^{n}$.

Clearly, we have $\mathcal{A}=\mathcal{C}[A]$ (as usual, by the symbol $\mathcal{C}[X]$ we denote the space of polynomials on $X$ with coefficients belonging to $\mathcal{C}$ ).

Let us prove that the variables generating $\mathcal{A}$ can be replaced by the variables generating $B$ and $C$. Indeed, $A_{n}=\widetilde{B}_{n} \oplus C_{n}$. Since $R_{i j k l ; I}-r_{i j k l ; I} \in \mathcal{C}\left[A^{n-1}\right]$ and $F_{i j \alpha ; I}-f_{i j \alpha ; I} \in \mathcal{C}\left[A^{n-1}\right]$ for $|I|=n-1$, we have

$$
\begin{aligned}
\mathfrak{C}\left[A^{n}\right] & =\mathcal{C}\left[A^{n-1} \oplus \widetilde{B}_{n} \oplus C_{n}\right]=\mathcal{C}\left[A^{n-1}\right] \otimes \mathcal{C}\left[\widetilde{B}_{n}\right] \otimes \mathfrak{C}\left[C_{n}\right] \\
& =\mathfrak{C}\left[A^{n-1}\right] \otimes \mathcal{C}\left[B_{n}\right] \otimes \mathcal{C}\left[C_{n}\right]=\prod_{k=0}^{n} \mathcal{C}\left[B_{k}\right] \otimes \mathcal{C}\left[C_{k}\right]=\mathcal{C}\left[B^{n}\right] \otimes \mathcal{C}\left[C^{n}\right]
\end{aligned}
$$

thus, $\mathcal{A}=\mathcal{C}[A]=\mathfrak{C}[B] \otimes \mathfrak{C}[C]$ as required.

Denote by $H^{n}$ the linear span $\mathcal{A}\left\langle\partial_{\mu} H, \partial_{\mu} \alpha_{\gamma}(|\mu| \leq N)\right\rangle$, where $\alpha=\alpha_{\gamma} e^{\gamma}$.

It can readily be shown that the expressions

$\delta \Gamma_{I}=-\partial_{I} H+\varphi,|I| \geq 3, \varphi \in H^{|I|-1}$,

$\delta \vartheta_{I ; \gamma}=-\partial_{I} \alpha_{\gamma}+\varphi,|\bar{I}| \geq 1, \varphi \in H^{|I|-1}$

$\delta x^{i}=\omega^{i j} \partial_{j} H$

$\partial_{p q} H$

$H-\langle\Omega, d H\rangle$, where $\Omega$ is a 1 -form such that $d \Omega=\omega$,

$\alpha_{\gamma}$

constitute a basis in $V$.

Let $W_{C}=\mathbb{R}\left\langle\delta \gamma_{I}, \delta \vartheta_{I \alpha}, \delta x^{i}\right\rangle$ and $W_{B}=\mathbb{R}\left\langle\partial_{p q} H, H-\langle\Omega, d H\rangle, \alpha_{\gamma}\right\rangle$.

Note that $R^{|I|}=R_{i j k l ; I}$ and $F^{|I|}=F_{i j \alpha ; I}$ are tensors; in other words, we have $s^{*}\left(R^{|I|}[\Gamma]\right)=R^{|I|}\left[s^{*} \Gamma\right]$ and $\sigma^{*}\left(F^{|I|}[\vartheta]\right)=F^{|I|}\left[\sigma^{*} \vartheta\right]$, where $\sigma$ is defined by (1). Hence,

$$
\delta R_{i j k ; I}, \delta F_{i j \alpha ; I} \in \mathbb{R}[B] \otimes W_{B} .
$$


Direct calculations show that

$$
\delta d x^{i}=\omega^{j i} \partial_{j p} H d x^{p} \in \mathbb{R}^{2 n} \otimes W_{B}, \quad \delta(H-\langle\Omega, d H\rangle)=0 .
$$

For every $\xi \in \mathfrak{s p}(2 n)^{*}$ and $\eta \in \mathfrak{g}^{*}$ we have

$$
\delta \xi\left(\partial^{2} H\right)=-\xi\left(\left[\partial^{2} H_{1}, \partial^{2} H_{2}\right]\right), \quad \delta \eta(\alpha)=-\eta\left(\left[\alpha_{1}, \alpha_{2}\right]\right) .
$$

Now we see that

$$
K_{C}^{\cdot}=\left(\mathcal{C}[C] \otimes \Lambda^{\cdot} W_{C}, \delta\right), \quad K_{B}^{\cdot}=\left(\mathbb{R}[B] \otimes \Lambda^{q} \mathbb{R}^{2 n} \otimes \Lambda^{\cdot} W_{B}, \delta\right)
$$

are complexes, and we have $\left(\mathcal{A}^{\cdot}, q, \delta\right)=\left(K_{C}^{\cdot} \otimes K_{B}^{\cdot}, \delta\right)$.

The complex $K_{C}$ is nothing other than the de Rham complex, and its cohomology is trivial except for $H^{0}\left(K_{C}\right)=\mathbb{R}$.

To calculate the cohomology of the complex $K_{B}$ we define an isomorphism $h: \mathfrak{m}^{*} \rightarrow W_{B} \subset \mathfrak{l}^{*}$ by the formula

$$
h(\xi \oplus \eta \oplus \zeta)=\xi\left(\partial^{2} H\right)+\eta(\alpha)+\zeta(H-\langle\Omega, d H\rangle) .
$$

We can readily verify (see (10) and (11)) that the mapping

$$
\delta: \mathbb{R}[B] \otimes \Lambda^{q} \mathbb{R}^{2 n} \rightarrow \mathbb{R}[B] \otimes \Lambda^{q} \mathbb{R}^{2 n} \otimes \mathfrak{m}^{*}, \quad \text { where } \mathfrak{m}^{*} \stackrel{h}{\cong} W_{C},
$$

defines a representation of the Lie algebra $m$ on $\mathbb{R}[B] \otimes \Lambda^{q} \mathbb{R}^{2 n}$, and $K_{B}^{*}$ is the cochain complex of this representation. Since the differential $\delta$ is trivial on $\mathbb{R} \subset \mathfrak{m}$ and $\mathfrak{s p}(2 n) \oplus \mathfrak{g}$ is a semisimple Lie algebra, we have

$$
H^{*}\left(K_{B}\right) \cong H^{0}\left(K_{B}\right) \otimes H^{*}(\mathrm{~m}) .
$$

To prove assertion (1) it remains to note that $H^{0}\left(K_{B}\right)=H^{0}\left(A^{0, \cdot}\right)=E_{1}^{0, \cdot}$.

To prove assertion (2) we note that $H^{*}(\mathfrak{m}) \stackrel{h_{*}^{*}}{\cong} H^{*}\left(\Lambda^{\cdot} W_{B}, \delta\right)$ and isomorphism (13) can be written in the form

$$
E_{1}^{\cdot,^{*}} \cong E_{1}^{0, \cdot} \otimes H^{\cdot}\left(W_{B}^{\cdot}\right)
$$

where $W_{B}^{\cdot} \subset E_{0}^{\cdot, 0}, H^{\cdot}\left(W_{B}^{\cdot}\right) \subset E_{1}^{\cdot, 0}$, and isomorphism (14) is generated by the multiplication in the algebra $E_{1}^{\cdot, \cdot}$. This completes the proof of the proposition.

Taking into account the assertions of Propositions 2 and 3, we can indicate the analogy between our spectral sequence and the spectral sequence of a universal bundle. To clarify this analogy, we consider the standard algebraic model of the spectral sequence of a universal bundle, namely, the spectral sequence of the Weil algebra, and define a morphism of the Weil algebra into $A^{*}$, .

Recall that by the Weil algebra associated with the Lie algebra $m$ we mean a graduated antidifferential algebra $W=S \mathrm{~m}^{*} \otimes \Lambda \mathrm{m}^{*}$, where $\mathrm{m}^{*}$ is the dual vector space of $\mathrm{m}$ and the gradation is given by the formula $W^{i}=\bigoplus_{2 m+k=i} S^{m} \mathfrak{m}^{*} \otimes \Lambda^{k} \mathfrak{m}^{*}$. The differential $d_{W}$ takes the following values on the generators of the algebra $W$ that have the form $1 \otimes \pi$ and $\pi \otimes 1$ with $\pi \in \mathrm{m}^{*}$ :

$$
d_{W}(1 \otimes \pi)=\pi \otimes 1+1 \otimes D \pi,
$$

where $D \pi \in \Lambda^{2} \mathrm{~m}^{*}$ and $D \pi(X, Y)=-\pi([X, Y])$ for $X, Y \in \mathfrak{m}$, and

$$
d_{W}(\pi \otimes 1) \in S^{1} \mathfrak{m}^{*} \otimes \Lambda^{1} \mathfrak{m}^{*}, \quad d_{W}(\pi \otimes 1)(X, Y)=\pi([X, Y]) .
$$

The filtration on $W$ has the form

$$
F^{p} W=\bigoplus_{2 m \geq p ; k} S^{m} \mathfrak{m}^{*} \otimes \Lambda^{k} \mathfrak{m}^{*}
$$

The spectral sequence of the algebra $W$ associated with this filtration is similar to the spectral sequence of a universal bundle. For more details concerning the Weil algebras and their spectral sequences see [4]. 
In our case we have $\mathfrak{m}=\mathfrak{s p}(2 n) \oplus \mathfrak{g} \oplus \mathbb{R}$. To define a morphism from $W$ into $A^{*}$, we introduce the variables $\Gamma_{k}=\left\|\Gamma_{k i j}\right\|_{i, j=1}^{2 n} \in \mathfrak{s p}(2 n), \Gamma=\Gamma_{k} d x^{k}$, where the elements of the Lie algebra $\mathfrak{s p}(2 n)$ are represented by symmetric $2 n \times 2 n$ matrices and the commutator is given by the formula $[a, b]_{i j}=$ $\omega^{p q}\left(a_{i p} b_{j q}+a_{j p} b_{i q}\right)$. Moreover, we assume that

$$
\vartheta_{i \alpha} d x^{i} e^{\alpha}=\vartheta_{i} d x^{i}=\vartheta \in \mathfrak{g} \otimes T^{*} U
$$

where $\left(e^{1}, e^{2}, \ldots\right)$ is a basis in $\mathfrak{g}$, and that

$$
\begin{gathered}
R_{i j}=\left\|R_{i j k l}\right\|_{k, l=1}^{2 n}=\frac{\partial \Gamma_{j}}{\partial x^{i}}-\frac{\partial \Gamma_{i}}{\partial x^{j}}+\left[\Gamma_{i}, \Gamma_{j}\right]_{\mathfrak{s p}(2 n)} \\
F_{i j}=F_{i j \alpha} e^{\alpha}=\frac{\partial \vartheta_{j}}{\partial x^{i}}-\frac{\partial \vartheta_{i}}{\partial x^{j}}+\left[\vartheta_{i}, \vartheta_{j}\right]_{\mathfrak{g}}
\end{gathered}
$$

are the curvatures of the connections $\nabla$ and $\nabla^{E}$. We set

$$
R=\frac{1}{2} R_{i j} d x^{i} \wedge d x^{j}, \quad F=\frac{1}{2} F_{i j} d x^{i} \wedge d x^{j} .
$$

The desired morphism $f: W \rightarrow A^{\cdot}$, is defined on the generators of $W$ as follows:

$$
\begin{aligned}
& f(1 \otimes(\xi \oplus \eta \oplus \zeta))=\xi\left(\partial^{2} H+\Gamma\right)+\eta(\alpha+\vartheta)+\zeta(H-\langle\Omega, d H\rangle+\Omega) \in A^{0,1} \oplus A^{1,0}, \\
& f((\xi \oplus \eta \oplus \zeta) \otimes 1)=\xi(R)+\eta(F)+\zeta(\omega) \in A^{0,2}
\end{aligned}
$$

where $\xi \in \mathfrak{s p}(2 n)^{*}, \eta \in \mathfrak{g}^{*}, \zeta \in \mathbb{R}, \xi \oplus \eta \oplus \zeta \in \mathfrak{m}^{*}, \partial^{2} H=\left\|\partial_{i j} H\right\| \in \mathfrak{s p}(2 n)$, and $\Omega$ is a 1-form such that $d \Omega=\omega$.

Note that $A=\bigoplus A^{p, q}$ is a filtered differential algebra with filtration (9), gradation $A^{r}=\bigoplus_{p+q=r} A^{p, q}$, and antidifferential $d+\delta$.

Proposition 6. The morphism $f$ is a morphism of filtered graded antidifferential algebras.

Proof. It suffices to verify that the filtration, gradation, and antidifferential are preserved for the generators of $W$. We can readily verify that the filtration and gradation are preserved. It remains to verify the relation $f d_{W}=(d+\delta) f$ for the generators of the algebra $W$.

We will prove only the relation $(d+\delta) f(1 \otimes \xi)=f\left(d_{W} 1 \otimes \xi\right)$ because in the other cases the calculations are similar.

By formulas (2)-(4), we have

$$
\begin{aligned}
d \xi\left(\partial^{2} H+\Gamma\right) & =-\xi\left(\partial^{3} H\right)+\xi\left(\partial \Gamma_{i} / \partial x^{j}\right) d x^{j} \wedge d x^{i} \\
& =-\xi\left(\partial^{3} H\right)+\xi\left(R_{j i}-\left[\Gamma_{j}, \Gamma_{i}\right]\right) d x^{j} \wedge d x^{i} / 2 \\
& =\xi\left(-\partial^{3} H+R-[\Gamma, \Gamma] / 2\right), \\
\delta \xi\left(\partial^{2} H\right)\left(X_{\alpha_{1}, H_{1}}, X_{\alpha_{2}, H_{2}}\right) & =L_{X_{H_{1}}} \xi\left(\partial^{2} H_{2}\right)-L_{X_{H_{2}}} \xi\left(\partial^{2} H_{1}\right)-\xi\left(\partial^{2}\left\langle\partial H_{1}, \partial H_{2}\right\rangle\right) \\
& =-\xi\left(\left[\partial^{2} H_{1}, \partial^{2} H_{2}\right]_{\mathfrak{s p}(2 n)}\right) \\
\delta \xi(\Gamma)\left(X_{\alpha, H}\right) & =L_{X_{H}} \xi(\Gamma)-\xi\left(\delta_{X_{\alpha, H}} \Gamma\right) \\
& =L_{X_{H}} \xi(\Gamma)-\xi\left(L_{X_{H}} \Gamma\right)+\xi\left(\partial^{3} H\right) \\
& =\xi\left(-\left[\partial^{2} H, \Gamma\right]+\partial^{3} H\right), \\
(d+\delta) f(1 \otimes \xi) & =(d+\delta) \xi\left(\partial^{2} H+\Gamma\right) \\
& =\xi\left(R-[\Gamma, \Gamma] / 2-\left[\partial^{2} H, \Gamma\right]-\left[\partial^{2} H_{1}, \partial^{2} H_{2}\right]\right), \\
f\left(d_{W} 1 \otimes \xi\right) & =f(\xi \otimes 1+1 \otimes D \xi) .
\end{aligned}
$$

Let $D \xi=\sum_{k} a_{k} \wedge b_{k}, a_{k}, b_{k} \in \mathfrak{s p}(2 n)^{*}$. 
It follows that

$$
\begin{aligned}
f\left(d_{W} 1 \otimes \xi\right) & =\xi(R)+\sum_{k} f\left(a_{k}\right) \wedge f\left(b_{k}\right) \\
& =\xi(R)+\sum_{k} a_{k}\left(\partial^{2} H+\Gamma\right) \wedge b_{k}\left(\partial^{2} H+\Gamma\right) \\
& =\xi(R)-\xi\left(\left[\partial^{2} H_{1}, \partial^{2} H_{2}\right]\right)-\xi\left(\left[\partial^{2} H, \Gamma\right]\right)-\xi([\Gamma, \Gamma] / 2) \\
& =(d+\delta) f(1 \otimes \xi) .
\end{aligned}
$$

This completes the proof of the proposition.

Let $\left(\mathcal{E}_{r}^{p, q}, d_{r}\right)$ be the spectral sequence of the filtered graded algebra $W$. Then the above proposition makes it possible to construct a morphism of multiplicative spectral sequences $f_{*}: \mathcal{E}_{r}^{p, q} \rightarrow E_{r}^{p, q}$ by means of which we reveal the structure of the terms $E_{2}^{p, q}$.

Proposition 7. (1) The graded algebras $E_{2}^{\cdot, 0}$ and $H^{\cdot}(\mathfrak{m})$ are isomorphic, and we have $E_{2}^{\cdot, 0}=f_{*}\left(\mathcal{E}_{2}^{\cdot, 0}\right)$.

(2) $E_{2}^{p, q} \cong E_{2}^{p, 0} \otimes E_{2}^{0, q}$, where the isomorphism is generated by the multiplication of factors on the right-hand side.

Proof. We first calculate $f_{*}\left(\mathcal{E}_{1}^{p, 0}\right)$. Note that $f_{*}$ induces an isomorphism of the complexes $\left(\mathcal{E}_{0}^{*, 0}, d_{W_{0}}\right)$ and $\left(W_{\dot{C}}^{\cdot}, \delta\right)$. Indeed, $\mathcal{E}_{0}^{p, 0}=1 \otimes \Lambda^{p} \mathfrak{m}^{*} \subset W / F^{1} W$ and $\left.f_{*}\right|_{\mathcal{E}_{0}^{p, 0}}=\left.\Lambda^{p} h\right|_{\Lambda^{p} \mathfrak{m}^{*}} \bmod F^{1}\left(A^{\cdot, \cdot}\right)$, where $h$ is isomorphism (12). Hence, $f_{*}$ is a bijective mapping of the groups $\mathcal{E}_{1}^{\cdot, 0}=H^{*}\left(\mathcal{E}^{\cdot, 0}, d_{0}\right)$ into the groups $H^{\cdot}\left(W_{C}^{\cdot}, \delta\right) \in E_{1}^{*, 0}$. It follows from Proposition 5 that

$$
E_{1}^{p, q}=E_{1}^{0, q} \otimes f_{*}\left(\mathcal{E}_{1}^{p, 0}\right)
$$

Since $\left.d_{1 W}\right|_{\mathcal{E}_{1}^{p, o}=0, \text { we have }}$

$$
\left.d_{1}\right|_{f_{*}\left(\varepsilon_{1}^{p, 0}\right)}=0 .
$$

Hence, $f_{*}\left(\mathcal{E}_{1}^{p, 0}\right)$ are not killed and $f_{*}\left(\mathcal{E}_{1}^{p, 0}\right) \subset E_{2}^{p, 0}$. Because $\mathcal{E}_{1}^{p, 0}=\mathcal{E}_{2}^{p, 0}$, for the corresponding images regarded as subspaces of $E_{2}^{p, 0}$ we have $f_{*}\left(\mathcal{E}_{1}^{p, 0}\right)=f_{*}\left(\mathcal{E}_{2}^{p, 0}\right)$.

By (17), we obtain

$$
E_{2}^{p, q}=E_{2}^{0, q} \otimes f_{*}\left(\mathcal{E}_{2}^{p, 0}\right) .
$$

Since $E_{2}^{0,0}=\mathbb{R}$, we have $E_{2}^{p, 0}=f_{*}\left(\mathcal{E}_{2}^{p, 0}\right)$ and $E_{2}^{p, q}=E_{2}^{0, q} \otimes E_{2}^{p, 0}$. This completes the proof of the proposition.

Now we can establish a closer analogy between the spectral sequences of the Weil algebra and of the bicomplex $A^{\cdot, \cdot}$.

Proposition 8. The mapping $f_{*}: \mathcal{E}_{r}^{p, q} \rightarrow E_{r}^{p, q}, r \geq 2$, is an isomorphism for $p+q<2 n$ and a monomorphism for $p+q=2 n$.

Proof. We apply the following standard lemma proved using dimensional considerations.

Lemma 2. Let $\left(\widetilde{E}_{r}^{p, q}, d_{r}\right)$ be a multiplicative spectral sequence such that

(a) $\widetilde{E}_{2}^{p, q}=\widetilde{E}_{2}^{p, 0} \otimes \widetilde{E}_{2}^{0, q}$

(b) the algebra

$$
\widetilde{E}_{2}^{*, 0}=\Lambda\left[c_{k}^{2 i-1}\left(i=1,2, \ldots ; k=1, \ldots, n_{i}\right)\right]
$$

is anticommutative and free and $\operatorname{dim} c_{k}^{2 i-1}=2 i-1$;

(c) $\widetilde{E}_{\infty}^{p, q}=0$ for $0<p+q<2 n$ and $\widetilde{E}_{\infty}^{0,0}=\mathbb{R}$.

Then

(1) for $i \leq n$ we have $d_{r} c_{j}^{2 i-1}=0, r<2 i$, and $d_{2 i} c_{j}^{2 i-1}=\tilde{c}_{j}^{2 i}$ in $\widetilde{E}_{2 i-1}^{0,2 i}$;

(2) let $c_{j}^{2 i}$ be a preimage of $\tilde{c}_{j}^{2 i}$ in $\widetilde{E}_{2}^{0,2 i}$; then the natural mapping

$$
F: \Lambda\left[c_{j}^{2 i-1}\left(i \leq n, j \leq n_{i}\right)\right] \otimes \mathbb{R}\left[c_{j}^{2 i}\left(i \leq n, j \leq n_{i}\right)\right] \rightarrow \widetilde{E}_{2}^{*, *},
$$


where $\operatorname{dim} c_{j}^{i}=i$, is an isomorphism in all dimensions less than $2 n$ and a monomorphism in the dimension $2 n$;

(3) $\widetilde{E}_{\infty}^{0,2 n}=\widetilde{E}_{2}^{0,2 n} / \operatorname{Im} F$.

For $n=\infty$ this lemma is known as the Zeeman theorem [6, p. 431].

Note that the sequence $\left(E_{r}^{p, q}, d_{r}\right)$ satisfies conditions (a) (Proposition 7), (b) (since $E_{2}^{*, 0} \cong f_{*}\left(\mathcal{E}_{2}^{*, 0}\right) \cong$ $H^{*}(\mathfrak{m})$, where $H^{*}(\mathfrak{m})$ is an algebra of the form (18)), and (c) (Proposition 3). The sequence $\varepsilon_{r}^{p, q}$ also satisfies these conditions. Because $\left.f\right|_{\varepsilon_{2}^{*}, 0}$ is an isomorphism and $f_{*} d_{W r}=d_{r} f_{*}$, it follows from Lemma 2(2) that $\left.f_{*}\right|_{E_{2}^{p, q}}$ is an isomorphism for $p+q<2 n$ and a monomorphism for $p+q=2 n$.

The proposition is proved.

We can now find the kernel of the mapping $E_{2}^{0, \cdot} \rightarrow E_{\infty}^{0, \cdot}$.

Corollary 1. $\operatorname{Ker}\left(E_{2}^{0, i} \rightarrow E_{\infty}^{0, i}\right)=f_{*}\left(\mathcal{E}_{2}^{0, i}\right)$.

Proof. For $i<2 n$ the assertion is a direct consequence of Proposition 8 ; for $i=2 n$ one has to apply Lemma 2(3).

To prove the theorem, it now suffices to note that $\mathcal{E}_{2}^{0, \cdot}$ is the space of invariant polynomials on $\mathfrak{m}$ and that the mapping $f_{*}$ just gives representation (6).

\section{References}

1. A. A. Abramov, "Topological invariants of Riemannian spaces obtained by integration of tensor fields," Dokl. Akad. Nauk SSSR, 81 (1951).

2. P. B. Gilkey, Invariance Theory, the Heat Equation, and the Atiyah-Singer Index Theorem, Math. Lect. Series, Vol. 11, Publish of Perish Inc., Wilmington, Del. (1984).

3. M. F. Atiyah, R. Bott, and V. K. Patody, "On the heat equation and the index theorem," Invent. Math., 19, 279-330 (1973).

4. D. B. Fuks, Cohomology of Infinite-Dimensional Lie Algebras [in Russian], Nauka, Moscow (1984).

5. P. Olver, Application of Lie Groups to Differential Equations, Springer-Verlag, New York-BerlinHeidelberg-Tokyo (1986).

6. R. M. Switzer, Algebraic Topology - Homotopy and Homology, Springer-Verlag, Berlin a.o. (1975).

Translated by A. I. Shtern 